\title{
A Hybrid Ant Colony Algorithm for Quadratic Assignment Problem
}

\author{
Guo Hong ${ }^{*}$
}

School of Software, Harbin University of Science and Technology, Harbin, China

\begin{abstract}
Quadratic assignment problem (QAP) is one of fundamental combinatorial optimization problems in many fields. Many real world applications such as backboard wiring, typewriter keyboard design and scheduling can be formulated as QAPs. Ant colony algorithm is a multi-agent system inspired by behaviors of real ant colonies to solve optimization problems. Ant colony optimization (ACO) is one of new bionic optimization algorithms and it has some characteristics such as parallel, positive feedback and better performances. ACO has achieved in solving quadratic assignment problems. However, its solution quality and its computation performance need be improved for a large scale QAP. In this paper, a hybrid ant colony optimization (HACO) has been proposed based on ACO and particle swarm optimization (PSO) for a large scale QAP. PSO algorithm is combined with ACO algorithm to improve the quality of optimal solutions. Simulation experiments on QAP standard test data show that optimal solutions of HACO are better than those of ACO for QAP.
\end{abstract}

Keywords: Quadratic assignment problem, Ant colony optimization, Particle swarm optimization, Optimal solutions, Simulation experiments.

\section{INTRODUCTION}

QAP is an NP-hard combinatorial optimization problem and it has been applied to many applications such as campus planning, hospital distribution, integrated circuits arrangement, job-shop scheduling and the selection of cooperative partners in virtual manufacturing circumstances [1]. It is difficult to find optimal solutions for QAP, when its scale is over 20. In order to overcome these difficulties in practice, one has to use approximation algorithms that do not guarantee an optimal solution but can find suboptimal solutions in a short time.

Ant colony optimization algorithm has been applied successfully to solve various combinatorial optimization problems as traveling salesman problem (TSP)[2], quadratic assignment problem [3], the shortest common supersequence problem and the resource-constraint project scheduling problem [4]. The principle of ACO is based on the way ants search for food and find their way back to the nest. Variants of ACO algorithms for the QAP have been proposed. Ant system is the first ACO algorithm which is designed to find optimal solutions for the QAP [5]. Thomas presents a maxmin ant system based on ant colony optimization, which exploits more strongly the best solutions found during the search, directs the ants' search towards solutions in high quality and avoids premature convergence of ants' search [6]. Bullnheimer proposes a parallel ant colony optimization algorithm where an information exchange between several colonies of ants is done every $\mathrm{k}$ generations [7]. Through simulation experiments, he shows how much the running

*Address correspondence to this author at the School of Software, Harbin University of Science and Technology, Harbin, China; Tel: 13836109611; Fax: 86397100; E-mail: guohong621@126.com time of the algorithm decreases with an increasing interval between the information exchange. Dorigo uses the cooperation between ants to find better solutions. Ants cooperate with each other by using an indirect form of communication mediated by pheromones they deposit on the edges of TSP graph when solutions are construceted [8]. Luca presents a new local optimizer called as SOP-3-exchange for the sequential ordering problem (SOP), in order to handle multiple constraints directly without increasing computational complexity. At the same time, SOP-3-exchange is combined with an ant colony optimization algorithm. The purpose is to improve the performance of the algorithm. Experimental results show that the new algorithm is more effective than existing methods for SOP [9]. ACO is an evolutionary algorithm for simulating behaviors that ant colony find foods. Positive feedback and heuristic information are applied to find optimal solutions. It has advantages such as parallel and high solving precision. Particle swarm optimization (PSO) is firstly intended for simulating social behaviors that a bird flock search for foods and it is a computational method that optimizes a problem by iteratively trying to improve a candidate solution with regard to a given measure of quality [10]. It is suitable to find the optimal combination of parameters in many algorithms and further optimize solutions searched by these algorithms.

In this paper, a hybrid ant colony algorithm is proposed for solving QAP. ACO algorithm is viewed as the main body and PSO algorithm is embodied into it. The purpose is to use PSO algorithm to improve ACO algorithm and make ACO algorithm not be lost in stagnation. Then some instances are extracted from QAPLIB. The hybrid ant colony algorithm and ant colony algorithm are respectively implemented on these instances. Experimental results show that performances of hybrid ant colony algorithm are better than those of ant colony algorithm. The rest of this paper is organized as fol- 
lows: quadratic assignment problem is described in section 2. Particle swarm optimization is described in section 3 , and hybrid ant colony algorithm is proposed in order to solve QAP in section 4. Experimental results are given in section 5. Conclusions of this paper are given in section 6 .

\section{THE DESCRIPTION OF QUADRATIC ASSIGN- MENT PROBLEM}

QAP is presented as a combinatorial optimization problem. It has been proven that QAP is a non-deterministic polynomial problem. There is no known algorithm for solving this problem in polynomial time, and even small instances may require long computation time. The traveling salesman problem may be seen as a special case of QAP if one assumes that the flows connect all facilities only along a single ring. QAP is described as follows: there are a set of $n$ facilities and a set of $n$ locations. For each pair of locations, a distance is specified. For each pair of facilities, a weight or flow is specified. For example, the amount of supplies transported between two facilities. The problem is to assign all facilities to different locations with the goal of minimizing the sum of distances multiplied by corresponding flows.

The formal definition of QAP is described as follows: the size of the facility set is $n$, the size of the location set is $n, d_{i j}$ is the distance between location $i$ and location $j$, and $f_{r s}$ is the flow between facility $r$ and facility s. Every facility will be assigned to a location. $M$ is an assignment plan. The cost function is defined in formula (1).

$F(M)=\sum_{i=1}^{n} \sum_{j=1}^{n} d_{i j} f_{M(i) M(j)}$

Here, M(i) is the facility assigned to location $\mathrm{i}$ and $\mathrm{M}(\mathrm{j})$ is the facility assigned to location $\mathrm{j}$. The optimization goal is to find an assignment plan $\mathrm{M}$, which makes the cost function $\mathrm{F}(\mathrm{M})$ minimized.

\section{THE DESCRIPTION OF PARTICLE SWARM OP- TIMIZATION}

PSO is firstly intended for simulating social behaviors that a bird flock search for foods and it has characteristics such as randomness, rapidity, global superiority and extensions. PSO optimizes a problem by having a population of candidate solutions, here called as particles, and moving these particles around in the search-space according to simple mathematical formulae over the particle's position and velocity. Each particle's movement is influenced by its local best known position and is also guided toward the best known positions in the search-space, which are updated as better positions found by other particles. This is expected to move the swarm toward the best solutions.

It is supposed that a bird flock find foods randomly in an area and all birds do not know locations of foods. But, they know distances between its current location and locations of foods. The simple and effective method is to search for areas of other birds nearest to foods. Heuristic information is used to find optimal solutions in PSO. In PSO, birds finding foods can be viewed as particles. Every particle has an adaptation value determined by the cost function and a velocity controlling its directions and distances. Other particles search for the solution space starting from the current optimal solution. Then optimal solutions can be found after many iterations. In each iteration, a particle updates itself with two extremums. One is the optimal solution found by this particle, called as an individual extremum. The other is the current optimal solution found by the swarm, called as a global extremum. Based on these two extremums, a particle updates its velocity and location with formula (2) and formula (3).

$$
\begin{aligned}
& V=\omega V+c_{1} r_{2}(\text { pBest }-X)+c_{2} r_{2}(\text { gBest }-X) \\
& X=X+V
\end{aligned}
$$

Here, $V=\left[v_{1}, v_{2}, \ldots, v_{d}\right]$ is velocity vector of particles, and $X=\left[x_{1}, x_{2}, \ldots, x_{d}\right]$ is current location vector of particles. $d$ is the dimension of the solution space. pBest is the individual extremum and gBest is the global extremum. $\omega$ is the weight. $c_{1}$ and $c_{2}$ are learning factors. $c_{1}$ is used to adjust the step distance, with which particles fly towards the best location. $c_{2}$ is used to adjust the step distance, with which particles fly towards the global best location. $r_{1}$ and $r_{2}$ are two independent random functions between 0 and 1 . Particles are updated iteratively and fly towards the location of the best solution. At last, gBest is the global best solution.

PSO has lots of advantages. The first one is that the algorithm is concise, has fewer parameters and is easy to be programmed. The second one is that the swarm is initialized and PSO has global searching ability. The third one is that the cost function is used to evaluate particles and the searching speed is fast. The fourth one is that its extension ability is good.

\section{THE HYBRID ANT COLONY ALGORITHM FOR QAP}

When ants find food, they will lay down pheromones on trails. Ants communicate with each other through pheromones. So they can cooperate with each other and finish complex tasks. In some areas, pheromones can attract ants and direct their actions. When some a trail gets marched over more frequently, the pheromone density becomes higher. The probability that more ants select this path is high and the pheromone density on this trail is strengthened. This is a positive feedback process and converges at the optimal path gradually. It is the principle of ant colony algorithms. In this paper, PSO algorithms are embedded into ACO algorithms for local optimization, so that its local searching ability and the optimal solution are improved.

Here, ant colony algorithm is applied to solve QAP. QAP may be viewed as the assignment plan of $n$ facility and $n$ location. Firstly, an unassigned facility is selected and it is assigned to a location according to selection rules. Then, the process is repeated until the problem solution is constructed by ants. Here, ants assign facility $\mathrm{i}$ to location $\mathrm{j}$ with some a rule according to random probability $q(0<q<1)$. This rule is shown as follows:

If probability $q$ is not over prior probability $q_{0}$, ant $\mathrm{k}$ will assign facility $i$ to location $j$ greedily according to the pheromone density and the visibility. It is described in formula (4).

$j=\max _{s \in \text { allowed }_{k}} \tau_{i s}^{\alpha} \cdot \eta_{i s}^{\beta}$ 
If probability $q$ is over prior probability $q_{0}$, ant $\mathrm{k}$ will assign facility $i$ to location $j$ with roulette wheel strategies. The probability $P_{i j}(t)$ assigning facility $\mathrm{i}$ to location $\mathrm{j}$ is computed according to formula (5). $P_{i j}(t)$ denotes the assignment probability between facility $\mathrm{i}$ and location $\mathrm{j}$ at time t. $P_{i j}(t)$ is described in formula(5).

$$
P_{i j}(t)=\left\{\begin{array}{ccc}
\frac{\tau_{i j}^{\alpha}(t) \cdot \eta_{i j}^{\beta}}{\sum_{s \in a l l o w e d} \tau_{k}^{\alpha}(t) \cdot \eta_{i s}^{\beta}} & \text { if } & j \in \text { allowed }_{k} \\
0 & \text { otherwise }
\end{array}\right.
$$

Here, allowed denotes the location set which ant k selects. $\alpha$ is heuristic factor, which describes the function of the deposited pheromone for ant actions. When the value of $\alpha$ is larger, the ant will be more apt to select other ants' trails. The cooperation degree between ants is higher. $\beta$ is the expected heuristic factor, and denotes the importance of the desirability. It describes the importance of heuristic information when ants select paths. When the value of $\beta$ is larger, state transition probabilities can be viewed as greedy rules. $\eta$ is the heuristic information between facility and location, which is computed by heuristic algorithms.

After every iteration, the left pheromone need be updated. The pheromone density between facility $i$ and location $\mathrm{j}$ can be adopted according to formula (6) and formula (7).

$\tau_{i j}(t+1)=(1-\rho) \tau_{i j}(t)+\rho \Delta \tau_{i j}(t)$

$\Delta \tau_{i j}(t)=\sum_{k=1}^{m} \Delta \tau_{i j}^{k}(t)$

Here, $\rho(1,0)$ means the evaporation degree of pheromones, and describes the decreasing degree of $\tau_{i j}(t) . \Delta \tau_{i j}(t)$ denotes the increase of pheromones between facility $i$ and location $\mathrm{j}$ at time $\mathrm{t}$ in this iteration. It is the pheromone density on trail $(\mathrm{i}, \mathrm{j})$ at time $\mathrm{t}$ in ACO. $\Delta \tau_{i j}^{k}(t)$ means pheromones left by ant $k$ between facility $i$ and location $j$ at time $t$ in this iteration. It is the pheromone density which ant $\mathrm{k}$ lays on trail $(i, j)$ at time $t$ in ACO.

In formula(4) and formula(5), pheromone density $\tau_{i j}$ denotes the priority that facility $\mathrm{i}$ is assigned to location $\mathrm{j}$. When the algorithm is initialized, it is set to $Q / L_{0}$. Here, $Q$ is a constant and $L_{0}$ is computed with the nearest neighbor assignment strategies. The principle of the nearest neighbor assignment strategies is that a minimum location is selected greedily for a facility. $\eta_{i j}$ is the heuristic value for assigning facility $i$ to location $j$, and its value is $1 /(0.1+C)$. When facility $\mathrm{i}$ is assigned to location $\mathrm{j}, C$ denotes the cost of facility $\mathrm{i}$ and others that are assigned before facility $i$ is assigned. $C$ is described in formula (8).

$C=\sum_{s \in t a b u_{k}} f_{s i} \cdot d_{u_{s} j}+f_{i s} \cdot d_{j u_{s}}$

Here, tabu, is a taboo table denoting the facility set assigned to ant k. $u_{s}$ is the location assigned to facility $s$.

The rule of updating pheromones globally is adopted here. After each iteration, the current optimal assignment strategy and the current optimal solution are used to update pheromones according to formula (7). Here, the value of $\Delta \tau_{i j}(t)$ is $Q / g$ BestLength. At the same time, $Q$ is a constant and gBestLength is the current optimal solution.

The hybrid ant colony algorithm is described as follows:

Step 1: Here, the number of particles and the number of ants are set to 50 . The weight of particles is set to 1 . The number of iterations in PSO is 20. Prior probability $q_{0}$ is set to 0.3 . Heuristic factor $\alpha$ is set to 1 . The expected heuristic factor $\beta$ is set to 2 . The evaporation degree of pheromones $\rho$ is set to 0.4 . Constant $Q$ is set to 100 and the number of iterations is 300 .

Step 2: If the current number of iterations is over the largest number of iterations, go to step 8 .

Step 3: Every ant constructs a new solution step by step according to selection rules.

Step 4: C is computed according to formula(8). Local searching algorithm is used to optimize the current solution. At the same time, the current solution and the assignment plan are modified.

Step 5: If all ants do not construct solutions, go to step 3. Otherwise, go to step 6.

Step 6: PSO is used to optimize local optimal solutions searched by all ants. At the same time, the assignment plan and the local optimal solution are modified.

Step 7: Based on the local optimal solution, the global optimal solution is found. According to the global optimal solution, the pheromone density is updated. Then, go to step 2. plan.

Step 8: Output the optimal solution and the assignment

\section{EXPERIMENTAL RESULTS AND ITS ANALYSIS}

QAPLIB is maintained by Rainer Burkard in order to provide a unified testbed for QAP [11], accessible to the scientific community. It consisted of virtually all QAP instances that are accessible to authors at any time. We select 4 instances from QAPLIB. They are respectively chr20c, kra30a, kra30b and lipa40b. Then ant colony algorithm and hybrid ant colony algorithm are implemented on these 4 instances for 20 times. Average values, optimal solutions and average iteration numbers of two algorithms are described in Table 1.

From Table 1, we can find that average values, optimal solutions and average iteration numbers of hybrid ant colony algorithm are better than those of ant colony algorithm. This is because that the optimization ability of ACO is improved after PSO is used. At the same time, its speed of global convergence is improved.

\section{CONCLUSIONS}

In this paper, we analyze characteristics of ant colony algorithm and particle swarm optimization algorithm. Particle swarm optimization algorithm is embodied into ant colony algorithm in order to improve its performance. At the same, simulation experiments are implemented on QAP standard 
Table 1. Experimental Results of Different QAPs on Two Algorithms

\begin{tabular}{|c|c|c|c|c|c|c|c|}
\hline \multirow{2}{*}{ Problems } & \multicolumn{3}{|c|}{ ACO } & \multicolumn{3}{c|}{ HACO } \\
\cline { 2 - 8 } & $\begin{array}{c}\text { Average } \\
\text { Values }\end{array}$ & $\begin{array}{c}\text { Optimal } \\
\text { Solutions }\end{array}$ & $\begin{array}{c}\text { Average Iteration } \\
\text { Numbers }\end{array}$ & $\begin{array}{c}\text { Average } \\
\text { Values }\end{array}$ & $\begin{array}{c}\text { Optimal } \\
\text { Solutions }\end{array}$ & $\begin{array}{c}\text { Average Iteration } \\
\text { Numbers } \\
\text { QAPLIB }\end{array}$ \\
\hline \hline chr20c & 14361.4 & 14142 & 429.7 & 14142 & 14142 & 42 & 14142 \\
\hline kra30a & 89942 & 88900 & 305.9 & 88900 & 88900 & 57.4 & 159.5 \\
\hline kra30b & 91512 & 91420 & 291.3 & 91467 & 91420 & 91420 \\
\hline lipa40b & 486257 & 478424 & 459.8 & 478518 & 478358 & 158.9 & 476581 \\
\hline
\end{tabular}

test data. Experimental results show that average values, optimal solutions and average iteration numbers of hybrid ant colony algorithm are better than those of fast ant colony algorithm. The performance of hybrid ant colony algorithm is better than that of ant colony algorithm.

\section{CONFLICT OF INTEREST}

The authors confirm that this article content has no conflicts of interest.

\section{ACKNOWLEDGEMENT}

Declared none.

\section{REFERENCES}

[1] T. Koopmans and M. Beckmann, "Assignment problems and the location of economics activities," Econometrica, vol. 25, pp.53-76, 1957.

[2] M. Dorigo, "The ant system: optimization by a colony of cooperating agents," IEEE Trans Syst. Man, Cybern, vol. 26, pp. 1-13, 1996.
[3] L. Maria Gambardella, "Ant colonies for the quadratic assignment problem," J. Oper. Res. Soc., vol. 50, pp. 167-176,1999.

[4] M. Middendorf, "Information exchange in multicolony ant algorithms," In: Proceedings of Third Workshop on Biologically Inspired Solutions to Parallel Processing Problems, Springer Verlag, London: UK pp. 645-652, 2000.

[5] V. Maniezzo, Alberto Colorni, and Marco Dorigo, "The ant system applied to the quadratic assignment problem," Technical Report IRIDIA/94-28, University Libre de Bruxelles, Belgium 1994.

[6] T. Stützle, "Max-min ant system," Future Generation Comput. Syst., vol. 16, pp. 889-914, 2000.

[7] B. Bullnheimer, and Gabriele Kotsis, "Parallelization strategies for the ant system," Appl. Optim., vol. 24, pp. 87-100,1998.

[8] M. Dorigo, and L. M. Gambardella, "Ant colony system: a cooperative learning approach to the traveling salesman problem," IEEE Trans. Evol. Comput., vol. 1, pp. 53-66,1997.

[9] L. M. Gambardella, and M. Dorigo, "An ant colony system hybridized with a new local search for the sequential ordering problem," INFORMS J. Comput., vol. 12, pp. 237-255, 2000.

[10] P-Y. Yin, Fred Glover, and Manuel Laguna, "A complementary cyber swarm algorithm," Int. J. Swarm Intell. Res., vol. 2, pp. 2241, 2011.

[11] R. Burkard, "Qaplib-a quadratic assignment problem library," Eur J. Oper. Res., vol.55, pp. 115-119, 1991.

(C) Guo Hong; Licensee Bentham Open.

This is an open access article licensed under the terms of the Creative Commons Attribution Non-Commercial License (http://creativecommons.org/licenses/by-nc/3.0/) which permits unrestricted, non-commercial use, distribution and reproduction in any medium, provided the work is properly cited. 